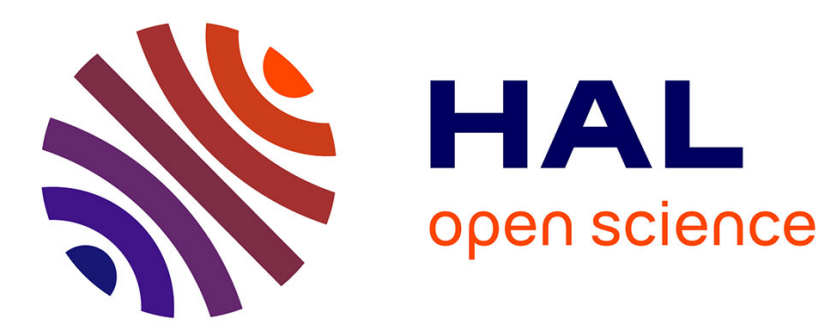

\title{
Étude de la butée des terres en présence d'écoulement
} Abdul-Hamid Soubra, Richard Kastner, Abdessamad Benmansour

\section{To cite this version:}

Abdul-Hamid Soubra, Richard Kastner, Abdessamad Benmansour. Étude de la butée des terres en présence d'écoulement. Revue Française de Génie Civil , 1998, 2 (6), pp.691-707. 10.1080/12795119.1998.9692200 . hal-01006810

\section{HAL Id: hal-01006810 https://hal.science/hal-01006810}

Submitted on 14 Mar 2017

HAL is a multi-disciplinary open access archive for the deposit and dissemination of scientific research documents, whether they are published or not. The documents may come from teaching and research institutions in France or abroad, or from public or private research centers.
L'archive ouverte pluridisciplinaire HAL, est destinée au dépôt et à la diffusion de documents scientifiques de niveau recherche, publiés ou non, émanant des établissements d'enseignement et de recherche français ou étrangers, des laboratoires publics ou privés. 


\section{Etude de la butée des terres en présence d'écoulement}

\section{Abdul-Hamid Soubra * - Richard Kastner ** Abdessamad Benmansour *}

\section{* LERGEC, ENSAIS}

24, Bd de la victoire, F-67084 Strasbourg cedex

Ahamid.Soubra@ensais.u-strasbg.fr

mansour@ligc.u-strasbg.fr

** URGC, INSA de Lyon

20, avenue Albert Einstein, F-69621 Villeurbanne cedex

Kastner@gcu-geot.insa-lyon.fr

Rlisumí. Cet article traite des effets liés à la mise hors d'eau d'excavations profondes. Une approche théorique est proposée pour évaluer le risque de renard et la diminution de la butée liée à l'écoulement. Le schéma de calcul retenu est basé sur la méthode cinématique de la théorie de l'analyse limite. Le mécanisme de rupture adopté est de type rotationnel. Il est constitué d'un bloc rigide délimité par une ligne de glissement en spirale logarithmique d'angle $\phi$. Les résultats numériques obtenus sont présentés et discutés.

ABSTRACT. This paper deals with the dewatering effects on deep excavations. A theoretical model is proposed to evaluate the risk due to the heaving phenomena and to compute the reduction of the passive earth pressures in the presence of seepage flow. The present calculation scheme is based on the upper-bound method of the limit analysis theory. The failure mechanism adopted is a rotational one. It is bounded by a log-spiral slip surface. The numerical results obtained are presented and discussed.

MOTS-CLES: pressions des terres, écoulement, renard, équilibre limite.

KEY WORDS: earth pressure, seepage, piping and heaving, limit state design. 


\section{Introduction}

L'exécution de travaux souterrains en site urbain conduit fréquemment à des excavations pénétrant profondément dans la nappe phréatique au sein d'aquifères de forte perméabilité.

L'écoulement souterrain entre l'amont et l'aval du rideau, lié à la mise hors d'eau est susceptible de provoquer une instabilité du fond de fouille : c'est le phénomène généralement dénommé « renard ». Il convient donc de considérer l'effet mécanique de l'écoulement induit par l'exhaure sur les poussées et butées des terres ainsi que sur la stabilité du fond de fouille. Ces divers effets sont souvent soit méconnus par les projeteurs, soit négligés, soit pris en compte de manière très approximative. Les phénomènes de réduction de la pression passive et du soulèvement en masse d'un prisme de sol devant la fiche sont en fait étroitement liés.

Après avoir rappelé les principales méthodes de prévision existantes, on présente une approche globale permettant d'évaluer la réduction de la pression passive en présence d'écoulement et de prévoir le risque de renard.

\section{Incidence mécanique de l'écoulement}

Les rabattements de nappe à l'abri d'un écran étanche induisent un écoulement sensiblement vertical ascendant devant la fiche. Les forces d'écoulement liées à ce flux ascendant diminuent le poids apparent du sol : il s'ensuit une réduction de la pression passive disponible devant la fiche. A la limite, ces forces peuvent provoquer des désordres couramment désignés sous le terme de renard. En fait, ce terme peut recouvrir deux phénomènes distincts : la boulance et le soulèvement généralisé (Kastner [KAS 82]).

- La boulance est un phénomène de surface qui survient lorsque le gradient hydraulique vertical y atteint sa valeur critique. Ce phénomène est souvent localisé en raison de l'hétérogénéité en perméabilité. Se développant progressivement, il peut être combattu par diminution du rabattement ou mise en place d'une surcharge filtrante.

- Le soulèvement généralisé est plus brutal : il concerne dans sa masse un prisme de sol devant la fiche susceptible de mettre en cause la stabilité de l'écran de soutènement.

\subsection{Prévision du risque de renard}

Terzaghi [TER 43] a introduit la notion de gradient critique $i_{c}=\gamma^{\prime} / \gamma_{w}$ conduisant, pour un écoulement unidirectionnel vertical, au soulèvement du sol, le poids déjaugé des grains étant compensé par les forces dues à l'écoulement. 
La boulance, phénomène de surface, éventuellement localisé, survient si, en un point du fond de fouille, le gradient hydraulique vertical atteint sa valeur critique. Cependant, les expériences de Marsland [MAR 53] et Kastner [KAS 82] ont montré qu'il pouvait y avoir soulèvement en bloc d'un prisme de sol devant la fiche, avant qu'il y ait boulance en surface. Divers auteurs ont examiné ce problème : Terzaghi [TER 43] propose de considérer l'équilibre d'un prisme EFIJ de largeur égale à f/2 et de hauteur variable (Figure 1). Tout en négligeant les forces de cisaillement le long des faces verticales du prisme, il considère l'équilibre de ce prisme soumis sur sa base à une distribution de pression interstitielle de résultante U. Faisant ensuite varier la hauteur du prisme, il retient le plus défavorable.

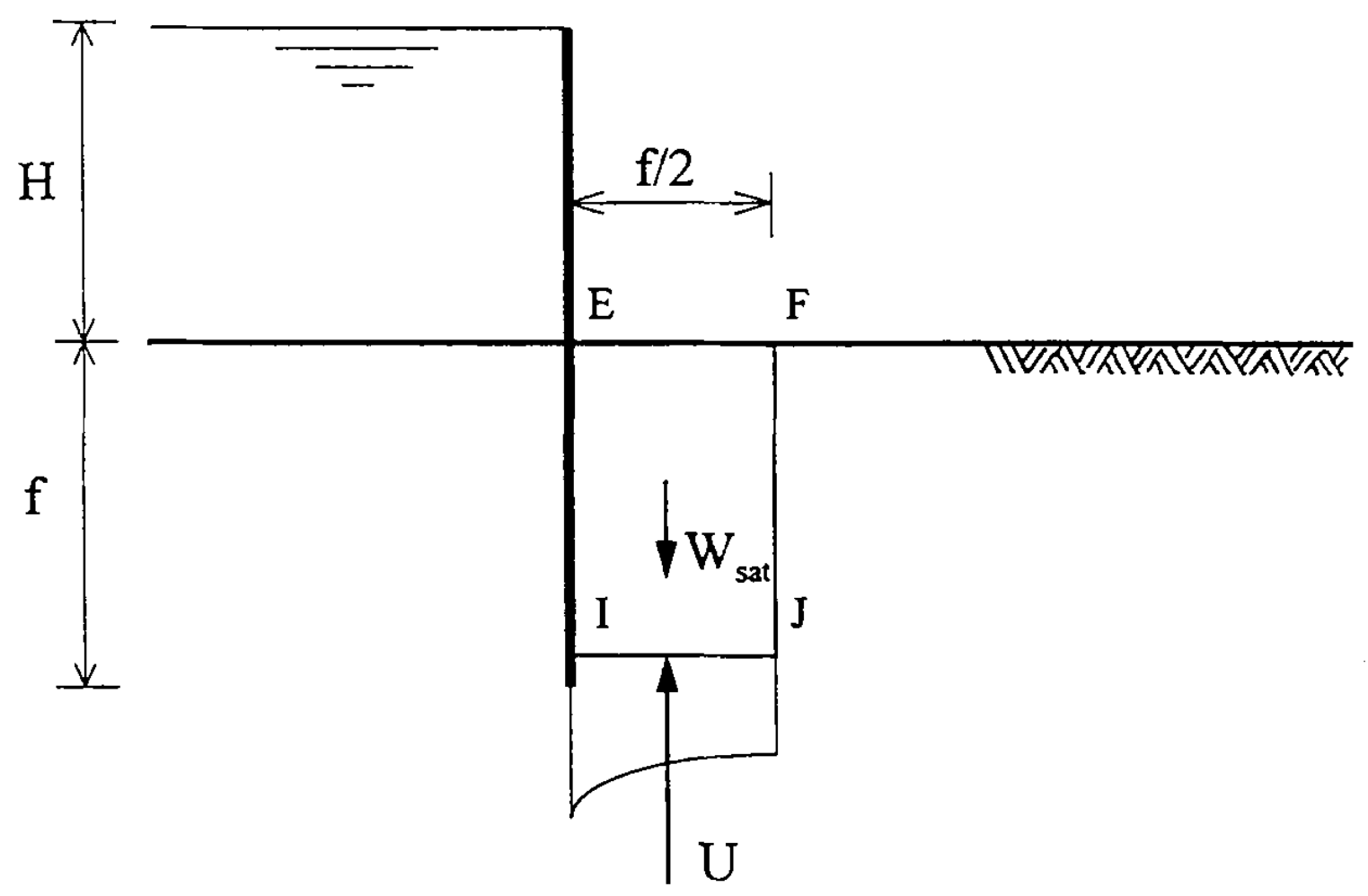

Figure 1. Schéma de rupture de Terzaghi. Terzaghi's failure mechanism

Davidenkoff [DAV 54] propose simplement de comparer le gradient moyen $i_{M}$ calculé sur la hauteur de la fiche au gradient critique.

Mandel [MAN 51] traite ce problème comme un problème de force portante de fondation : après avoir calculé les contraintes effectives externe et interne au pied de l'écran, il considère le sol externe comme une charge de fondation. Cette approche qui néglige les contraintes de cisaillement sur les faces verticales des prismes en rupture, tant pour le sol externe que pour le sol interne apparaît parfois bien trop pessimiste.

Enfin, Bazant [BAZ 63] prend en compte les frottements en considérant un prisme de rupture circulaire se développant à partir du pied de l'écran sous l'effet des forces d'écoulement. 
La comparaison de ces différentes approches dans le cas d'un écran fiché dans un milieu semi-infini fait apparaître des écarts importants pouvant atteindre $75 \%$ pour la charge d'eau provoquant la rupture [KAS 82].

\subsection{Prise en compte du risque de renard et de la diminution de la pression passive}

Les différentes méthodes proposées dans la littérature reposent sur des hypothèses assez restrictives, notamment pour le choix du mécanisme de rupture et la prise en compte de la résistance au cisaillement du sol. Par ailleurs, ces méthodes ne prennent en compte que le rôle de coupure étanche des écrans, alors que ceux-ci ont fréquemment un rôle simultané d'écran étanche et de soutènement. Dans ces conditions, la fiche est en appui sur le sol en fond de fouille et mobilise partiellement sa résistance pour assurer l'équilibre global du rideau de soutènement.

Enfin, les expériences de Marsland confirmées par celles de Kastner ont fait apparaître des prismes de rupture délimités par une courbe passant par le pied de l'écran, et non un prisme de rupture rectangulaire. Ces observations nous ont amené à proposer un modèle de calcul de la butée qui peut être mobilisée devant un rideau de palplanches en présence d'un écoulement hydraulique.

\section{Schéma de calcul de la pression passive des terres en présence d'écoulement}

Le problème de butée en présence d'écoulement est envisagé grâce à l'étude de la stabilité d'un volume du sol en rupture $\mathrm{OAB}$ situé à l'aval de la paroi (Figure 2). La résultante $\mathrm{P}_{\mathrm{pe}}$ des pressions passives effectives agissant sur ce volume du sol est représentée par une seule force inclinée d'un angle $\delta$ (angle de frottement sol-paroi) par rapport à la normale à la paroi, et dont le point d'application est situé au tiers inférieur de la fiche. Dans cette analyse, la force $\mathrm{P}_{\mathrm{pe}}$ est considérée comme un chargement extérieur au volume du sol en glissement. D'autres analyses possibles consistent à considérer un volume de sol dont la frontière passe sous la paroi et débouche sur la surface libre en amont de l'ouvrage. Dans cette dernière analyse, la force $\mathrm{P}_{\mathrm{pe}}$ est considérée comme un effort interne au système sol-paroi. Cependant, ce type de rupture n'est possible que lorsque l'ouvrage lui-même est très fortement étayé avec une fiche très courte. Ce cas ne correspond pas au problème étudié car la butée n'intervient pas dans la stabilité de l'ouvrage.

Pour la prise en compte des forces d'écoulement dans l'étude du problème de butée, deux approches équivalentes sont possibles : soit, on applique au poids déjaugé du prisme de sol en rupture, des forces de volume proportionnelles en tout point aux gradients hydrauliques, soit on considère le poids saturé de la masse de sol en rupture, soumise à la résultante des pressions interstitielles appliquées sur son contour. Dans l'analyse présente, nous avons choisi d'utiliser la deuxième approche (Figure 2). 


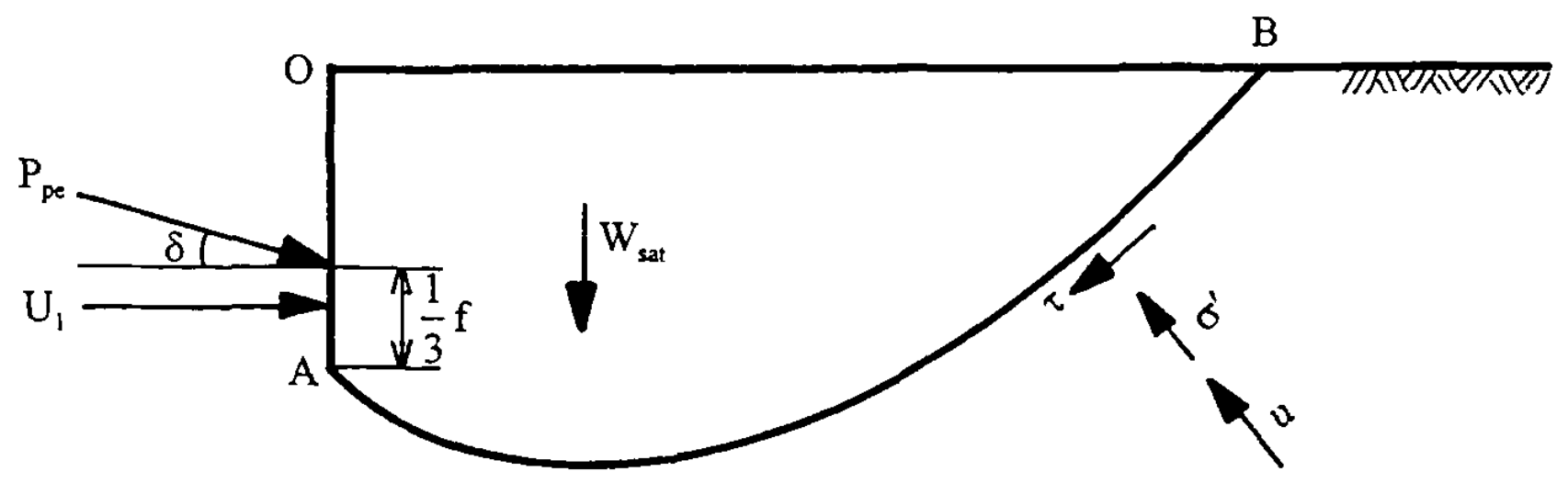

Figure 2. Prisme de rupture en butée. Passive failure wedge

\subsection{Hypothèses}

Les hypothèses prises en compte dans cette étude sont :

- le sol considéré dans la présente analyse est constitué d'un matériau purement frottant de caractéristiques mécaniques constantes, mais de propriétés hydrauliques variables. Il est donc caractérisé par son angle de frottement interne $\phi$, son poids volumique saturé $\gamma_{\mathrm{sat}}$ et ses coefficients de perméabilité $\mathrm{K}_{\mathrm{i}}$ où $\mathrm{K}_{\mathrm{i}}$ représentent les coefficients de perméabilité isotrope des différentes couches. De telles configurations se rencontrent dans le cas des dépôts alluvionnaires sablo-graveleux qui se présentent souvent sous forme de strates plus ou moins sableuses. Si les propriétés mécaniques de ces différents horizons sont sensiblement constantes, leur perméabilité peut être très fortement affectée par des variations de granulométrie ;

- on considère que le sol a une perméabilité suffisamment élevée pour que sa consolidation soit quasi immédiate. Les déformations du massif de sol peuvent donc être négligées. L'écoulement pouvant dès lors être considéré comme permanent, il n'y a pas d'incidence de la déformation du milieu granulaire sur les pressions interstitielles.

\subsection{Méthode utilisée}

L'étude des problèmes de stabilité par la théorie de l'analyse limite (Méthodes cinématique et statique) a fait l'objet de nombreux travaux. On peut citer entre autres les travaux de [SAL 90, 93], [DEB 92, 93], [PAS 78], [BOT 80] et [SOU 92, 97, 98]. Dans ce travail, notre choix s'est porté sur l'approche cinématique de la théorie de l'analyse limite. Cette méthode consiste à choisir un mécanisme de rupture cinématiquement admissible respectant les conditions aux limites en vitesse, et à égaler la puissance des efforts extérieurs à la puissance dissipée [SAL 83]. Le mécanisme de rupture retenu dans la présente analyse est délimité par une surface de glissement en spirale logarithmique. Il correspond à la rotation d'un bloc rigide autour du foyer de la spirale logarithmique avec une vitesse angulaire $\dot{\Omega}$ (Figure 3 ). 


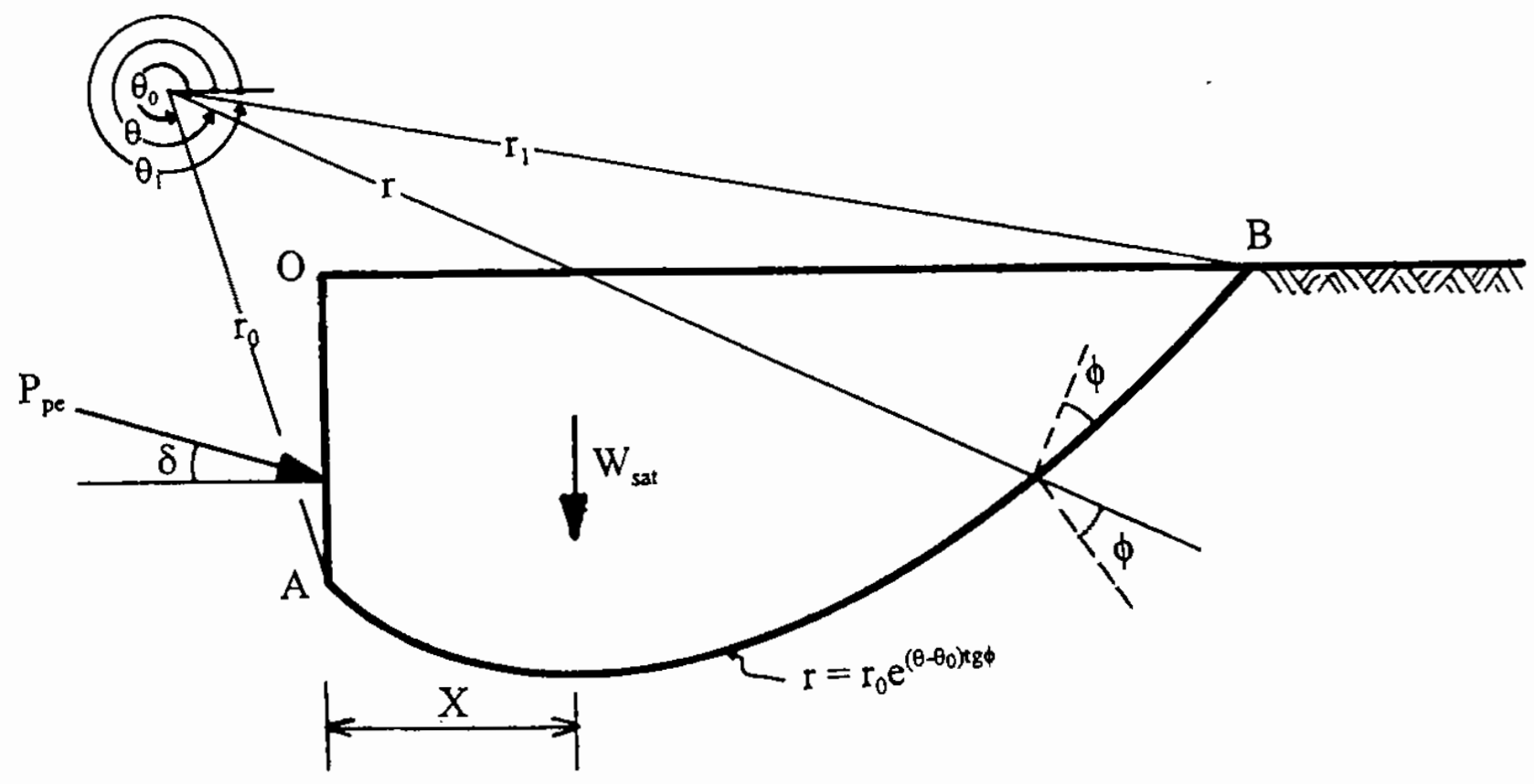

Figure 3. Mécanisme de rupture. Failure mechanism

Le choix de ce mécanisme est guidé par des études antérieures. En effet, Soubra [SOU 89] et Soubra et al. [SOU 98] ont appliqué l'approche variationnelle à la méthode du prisme de rupture. Cette approche a permis d'aboutir à la forme de la surface de rupture et à la distribution des contraintes normales effectives agissant le long de cette surface pour lesquelles les trois équations de l'équilibre statique de la masse en glissement sont vérifiées et la charge de rupture correspondante est minimale. Ces auteurs ont montré que la forme de la surface de rupture est une spirale logarithmique d'angle $\phi$ et que la distribution des contraintes normales effectives agissant le long de la ligne de glissement n'est pas nécessaire au caicul de la charge de rupture : il suffit d'écrire l'équation d'équilibre des moments de tous les efforts agissant sur le prisme de rupture par rapport au foyer de la spirale logarithmique pour calculer la charge de rupture. Enfin, ces auteurs ont montré qu'il existe une équivalence entre la méthode du prisme de rupture variationnelle et la méthode cinématique de la théorie de l'analyse limite pour un mécanisme rotationnel délimité par une spirale logarithmique. Pour plus de détail, le lecteur pourra se référer à d'autres publications (Soubra et al. [SOU 98]). Cette méthodologie a également été utilisée par plusieurs auteurs dans d'autres domaines de stabilité en géotechnique. On peut citer notamment les travaux de Baker et Garber [BAK 78] et ceux de Leshchinsky et al. [LES 85] dans l'étude de la stabilité des talus. Par ailleurs, ce mécanisme rotationnel a également été utilisé par d'autres auteurs dans le cadre de la théorie de l'analyse limite (Chen [CHE 75] ; Anthoine [ANT 90] ; De Buhan et al. [DEB 92]) pour l'étude de la stabilité des pentes. Les résultats obtenus par ces auteurs montrent la pertinence du choix de ce mécanisme de rupture. 


\subsection{Principe du calcul}

Le principe du calcul de la butée adopté dans cet article est donc basé sur l'équation du bilan énergétique du mécanisme de rupture adopté (Figure 3). L'équation du bilan énergétique, après avoir simplifié la vitesse angulaire $\dot{\Omega}$ s'exprime :

$\mathrm{W}_{\text {sat }}\left(\mathrm{r}_{0} \cos \theta_{0}+\mathrm{X}\right)+\mathrm{P}_{\mathrm{P}_{\mathrm{e}}} \sin \delta\left(\mathrm{r}_{0} \cos \theta_{0}\right)=\mathrm{P}_{\mathrm{P}_{\mathrm{e}}} \cos \delta\left(-\mathrm{r}_{0} \sin \theta_{0}-\frac{\mathrm{f}}{3}\right)+\mathrm{M}_{1}+\mathrm{M}_{2}$

où $M_{1}$ et $M_{2}$ représentent les moments, par rapport au centre de la spirale logarithmique, des pressions interstitielles agissant respectivement le long de la fiche et le long de la ligne de glissement.

L'équation (1) montre que $P_{p e}$ est fonction des deux paramètres angulaires $\theta_{0}$ et $\theta_{1}$ décrivant le mécanisme de rupture. La valeur du coefficient de butée $\mathrm{K}_{\text {pye }}$ $\left(\mathrm{K}_{\mathrm{pye}}=2 \mathrm{P}_{\mathrm{Pe}} / \gamma^{\prime} \mathrm{f}^{2}\right)$ la plus critique est obtenue en minimisant ce coefficient par rapport aux deux paramètres angulaires $\theta_{0}$ et $\theta_{1}$. Un code de calcul a été développé pour déterminer les valeurs minimales du coefficient de butée et les surfaces de rupture critiques correspondantes.

\section{Résultats numériques}

\subsection{Butée en l'absence d'écoulement}

Nous présentons dans le tableau (1), les résultats du coefficient de butée obtenus par treize auteurs dans le cas où $\phi=40^{\circ}$ et $\delta=20^{\circ}$.

\begin{tabular}{||l|c|}
\hline Auteurs & Valeurs de $\boldsymbol{K}_{p \gamma}$ \\
\hline \hline Coulomb (1776) & 11.77 \\
\hline Terzaghi (Packshaw, 1946) & 10.82 \\
\hline Terzaghi (Shields et Tolunay, 1972) & 9.66 \\
\hline Shields et Tolunay (1973) & 8.30 \\
\hline Rahardjo et Fredlund (1984) & 9.63 \\
\hline Habibagahi et Ghahramani (1979) & 9.95 \\
\hline Solution présente & 9.81 \\
\hline Caquot et Kérisel (1948) & 9.60 \\
\hline Sokolovski (1960) & 9.68 \\
\hline Lysmer (1970) & 9.54 \\
\hline Basudhar et al. (1979) & 9.34 \\
\hline Lee et Heringtoon (1972) & 9.30 \\
\hline Chen et Rosenfarb (1973) & 10.10 \\
\hline
\end{tabular}

Tableau 1. Valeurs du coefficient de butée proposées par différents auteurs pour $\phi=40^{\circ}$ et $\delta=20^{\circ}$. Values of the passive earth pressure coefficient as proposed by different authors for $\phi=40^{\circ}$ and $\delta=20^{\circ}$ 
Ce couple $(\phi, \delta)$ a été retenu d'une part parce qu'il correspond à un cas fréquemment étudié, d'autre part parce qu'il fait apparaître des divergences notables entre les diverses méthodes, divergences qui sont gommées lorsque $\phi$ et surtout $\delta$ sont plus faibles. Nous avons retenu comme référence les tables de Caquot et Kérisel [cf. Kérisel et Absi, 1990]. Si l'on examine maintenant les résultats obtenus par les méthodes de prisme de rupture, il apparaît qu'ils sont assez dispersés : certains dépassent les valeurs de Caquot et Kérisel par excès (Coulomb) ; d'autres par défaut (Shields et Tolunay [SHI 73]). Ceci est bien évidemment dû i) aux hypothèses concernant le choix a priori de la forme de la ligne de glissement et de la distribution des contraintes normales le long de cette ligne et ii) au fait que cette catégorie de méthodes ne respecte pas toutes les équations de l'équilibre statique global de la masse en glissement. La solution présente $\left(\mathrm{K}_{\mathrm{pY}}=9,81\right)$ est très proche de celle donnée par les tables de Caquot et Kérisel, l'écart est de l'ordre de $2 \%$.

Afin de comparer nos résultats sur une plus grande gamme de couples $(\phi, \delta)$, nous présentons dans la figure 4 et dans le tableau 2 les résultats donnés par Caquot et Kérisel montrant la variation de $\mathrm{K}_{\mathrm{py}}$ en fonction de $\phi$ pour deux cas différents du rapport $\delta / \phi(\delta / \phi=-1 / 3 ;-1)$. Nous présentons également les résultats obtenus par l'approche présente pour les mêmes cas.

Les courbes correspondant au cas où $\delta / \phi=-1 / 3$ montrent que les résultats obtenus par la présente méthode sont pratiquement identiques à ceux obtenus par Caquot et Kérisel. Par contre, pour $\delta / \phi=-1$, les résultats sont identiques pour les faibles valeurs de $\phi$, puis l'écart augmente au fur et à mesure de l'augmentation de $\phi:$ il atteint $10 \%$ à $\phi=40^{\circ}$. Ainsi, pour les valeurs que l'on rencontre couramment dans la pratique $\phi \leq 40^{\circ} ; \delta / \phi \geq-2 / 3$, l'écart maximum entre les valeurs de Caquot et Kérisel et celles de notre approche ne dépasse pas $5 \%$ et devient négligeable pour $\phi \leq 35^{\circ}$.

\begin{tabular}{||c|c|c||c|c|}
\hline \multirow{3}{*}{$\phi\left({ }^{\circ}\right)$} & \multicolumn{2}{|c|}{$\delta / \phi=-1$} & \multicolumn{2}{c|}{$\delta / \phi=-1 / 3$} \\
\cline { 2 - 5 } & Caquot et Kérisel & Solution présente & Caquot et Kérisel & Solution présente \\
\hline 10 & 1.66 & 1.67 & 1.52 & 1.51 \\
\hline 15 & 2.2 & 2.25 & 1.89 & 1.88 \\
\hline 20 & 3.04 & 3.13 & 2.38 & 2.39 \\
\hline 25 & 4.26 & 4.54 & 3.03 & 3.07 \\
\hline 30 & 6.56 & 6.93 & 4.02 & 4.03 \\
\hline 35 & 10.7 & 11.3 & 5.55 & 5.44 \\
\hline 40 & 18.2 & 20.01 & 8.1 & 7.61 \\
\hline 45 & 35 & 39.48 & 12 & 11.18 \\
\hline
\end{tabular}

Tableau 2. Valeurs du coefficient de butée données par l'approche présente et par Caquot et Kérisel. Values of the passive earth pressure coefficient as given by the present analysis and that of Caquot and Kérisel 


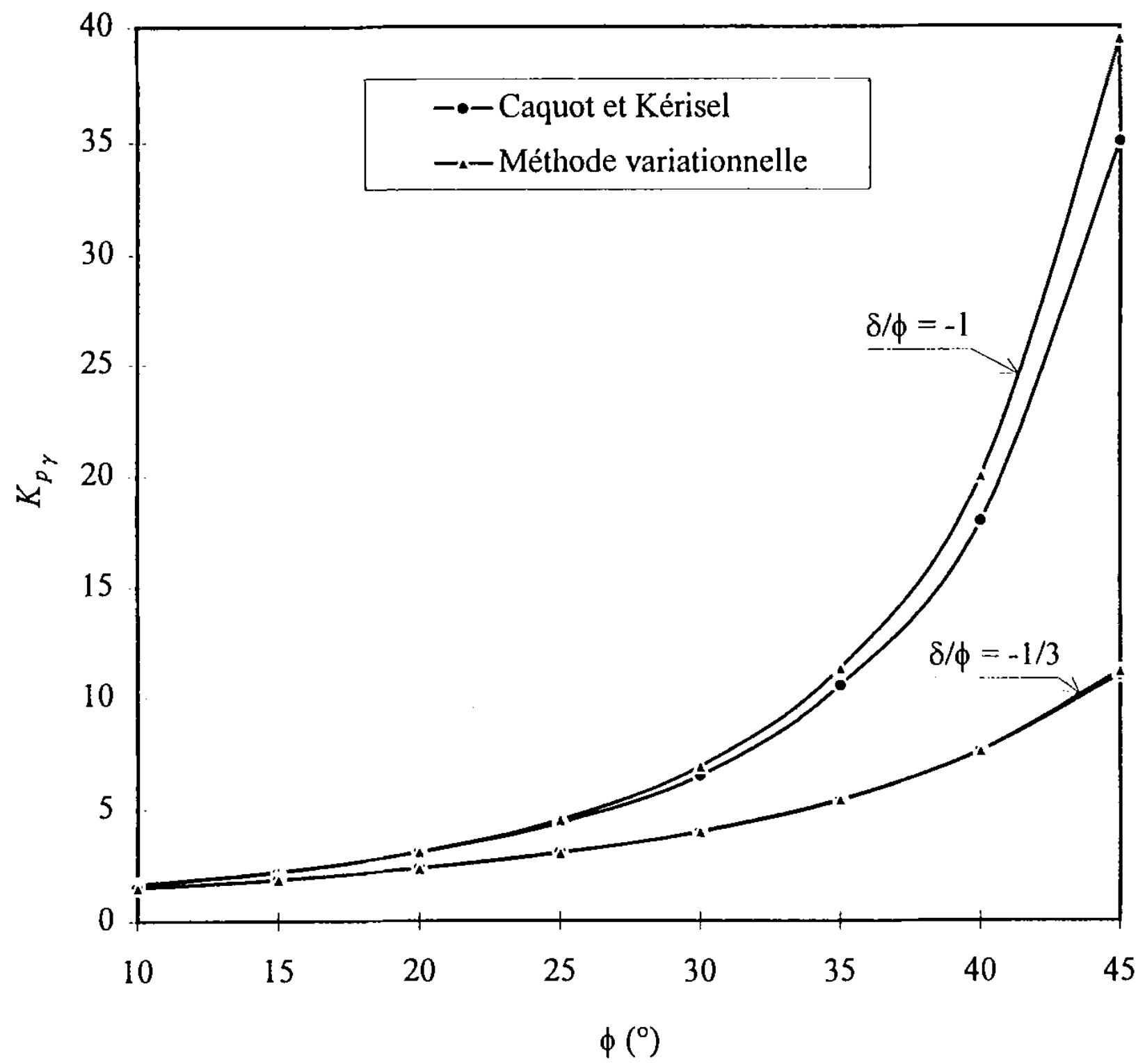

Figure 4. Comparaison entre la solution présente et celle de Caquot et Kérisel. Comparison between the present solution and that of Caquot and Kérisel

\subsection{Butée en présence d'écoulement}

Les sols réels étant souvent fortement anisotropes et hétérogènes du point de vue de leur perméabilité, nous nous sommes intéressés au cas d'un batardeau de largeur finie fiché dans un sol multicouche en ce qui concerne la perméabilité (Figure 5).

Dans le cas général, la distribution des pressions interstitielles ne peut être obtenue analytiquement et nécessite un traitement numérique. Nous avons choisi d'utiliser la méthode des différences finies pour la résolution de l'équation de l'écoulement. Cette méthode a permis de donner la distribution des charges hydrauliques et des pressions interstitielles aux nœuds du maillage considéré. Les conditions aux limites considérées dans cette étude sont données sur la figure 6, où l'on fait l'hypothèse 
d'une alimentation latérale vers le batardeau à une distance $\mathrm{L}$ du rideau. Dans la suite de cet article, la demi largeur du batardeau $\mathrm{B}_{0} / 2$ est fixée à $10 \mathrm{~m}$.

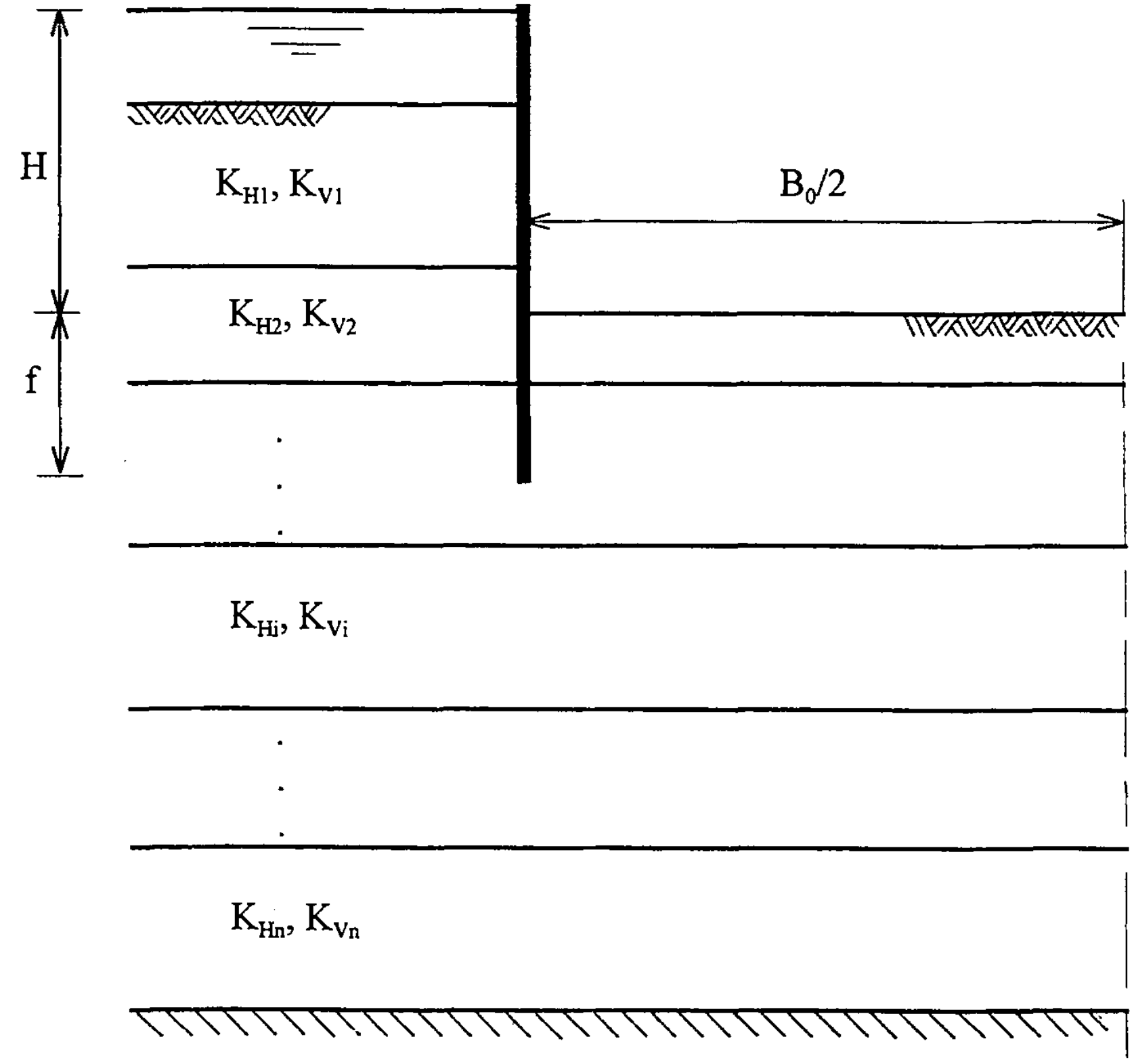

Figure 5. Cas d'un batardeau filant fiché dans un sol multicouche. Case of a strip cofferdam in a multi layered soil medium

Les moments $M_{1}$ et $M_{2}$ de l'équation (1) ont été déterminés numériquement en utilisant la méthode d'intégration de Gauss. La détermination des pressions interstitielles le long de la spirale logarithmique ainsi que le long de la fiche a été effectuée à partir des pressions calculées aux nœuds du maillage en utilisant une interpolation bilinéaire.

Dans la suite de cet article, nous allons présenter quelques résultats concernant la variation de la butée en présence d'écoulement dans le cas d'un batardeau fiché dans un sol hétérogène en ce qui concerne la perméabilité. Tous les résultats qui suivent considèrent comme poids volumique saturé du sol : $\gamma_{\mathrm{sat}}=20 \mathrm{kN} / \mathrm{m}^{3}$ et concernent le cas d'une alimentation latérale située à $\mathrm{L}=60 \mathrm{~m}$ à partir du rideau. 


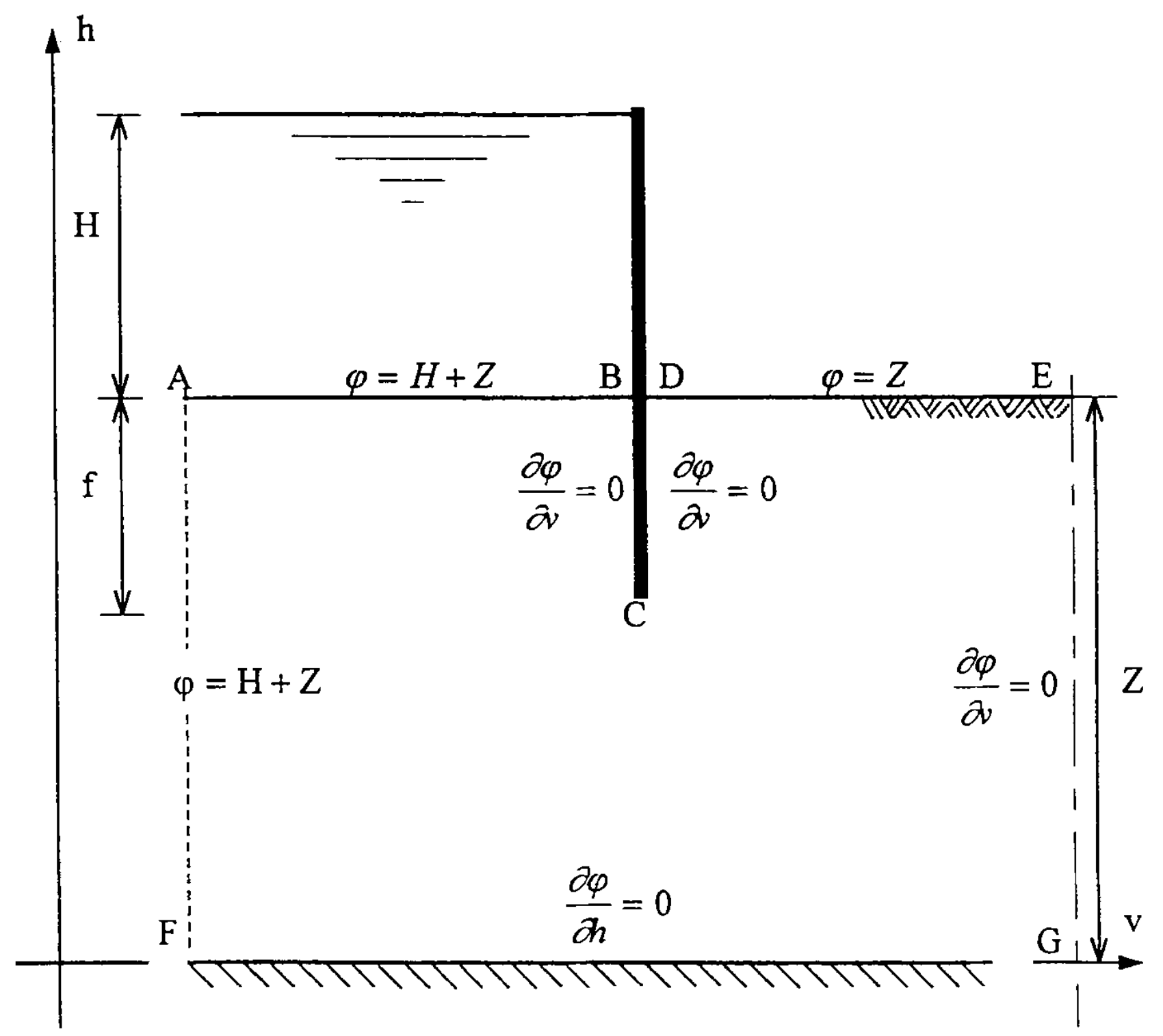

Figure 6. Conditions aux limites de l'écoulement. Boundary conditions of the seepage flow

\subsubsection{Batardeau fiché dans un sol tricouche}

A titre d'exemple, on examine le cas d'un sol tricouche, l'écran étant fiché à mihauteur dans la couche intermédiaire. Le coefficient de perméabilité des couches extrêmes est identique et est noté $\mathrm{K}_{1}$, et celui de la couche intermédiaire est noté $\mathrm{K}_{2}$ (Figure 7).

Nous présentons sur la figure 8 les résultats de la variation du coefficient de butée en présence d'écoulement en fonction du rapport $\mathrm{H} / \mathrm{f}$ dans le cas où $\mathrm{K}_{1} / \mathrm{K}_{2}=5, \phi=35^{\circ}$ et $\delta / \phi=-1 / 3 ;-1 / 2 ;-2 / 3$.

Notons que la réduction du coefficient de butée est quasi linéaire. On s'aperçoit que pour une butée nulle, l'angle de frottement sol-structure n'a pas d'influence sur la valeur de $\mathrm{H} / \mathrm{f}$ provoquant la rupture. En effet, lorsque la butée est nulle, il n'y a plus d'interaction entre la fiche et le sol ; il s'agit du soulèvement en masse tel qu'il est 
envisagé traditionnellement. Par contre, dès que $\mathrm{H} / \mathrm{f}$ diminue et qu'il apparaît ainsi une force de butée, le résultat est bien fonction de l'angle de frottement sol-écran $\delta$.

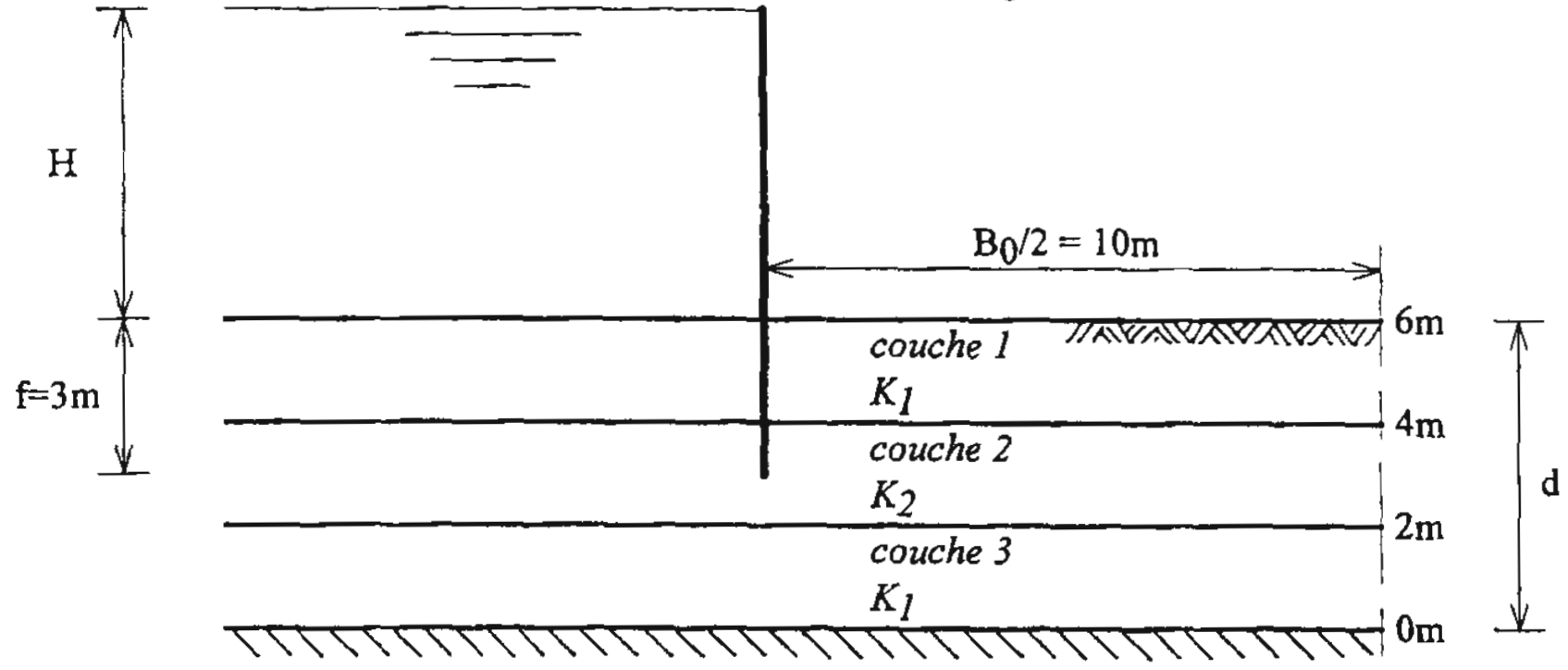

Figure 7. Cas d'un batardeau fiché dans un sol tricouche. Case of a cofferdam in a three layered soil medium

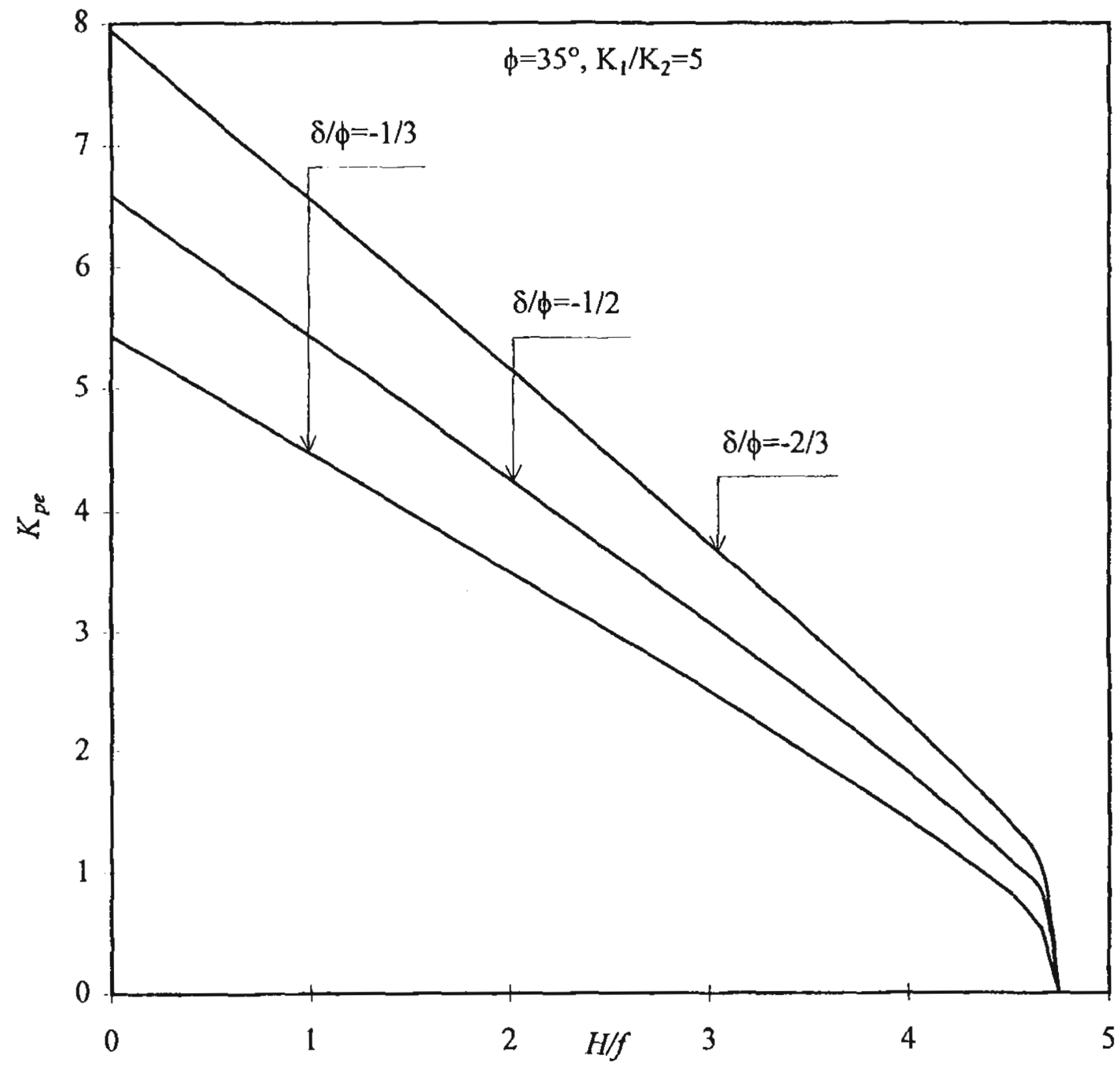

Figure 8. Variation de Kpe en fonction de H/f. Variation of Kpe with $\mathrm{H} / \mathrm{f}$ 
Afin d'étudier l'effet du rapport de perméabilité $\mathrm{K}_{1} / \mathrm{K}_{2}$ sur le coefficient de butée, nous présentons sur la figure 9 la variation du coefficient de butée avec $\mathrm{K}_{1} / \mathrm{K}_{2}$ quand $\phi=35^{\circ}, \delta / \phi=-2 / 3$ et $\mathrm{H} / \mathrm{f}=2$.

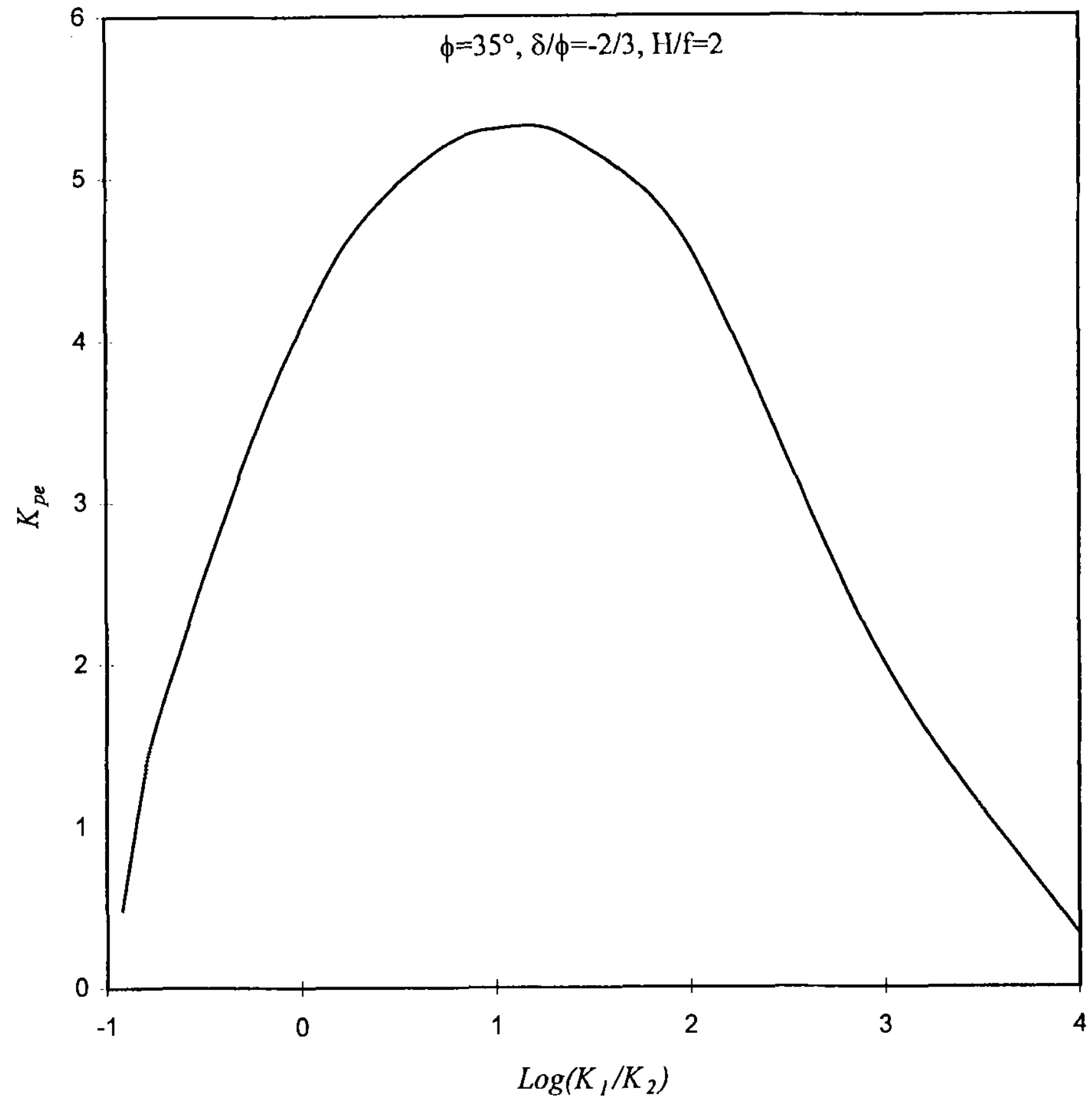

Figure 9. Variation de $\mathrm{K}_{\mathrm{pe}}$ avec le rapport de perméabilité $\mathrm{K}_{1} / \mathrm{K}_{2} . \mathrm{K}_{\mathrm{pe}}$. Values versus permeability ratio $\mathrm{K}_{1} / \mathrm{K}_{2}$

Pour un sol homogène $\left(\mathrm{K}_{1} / \mathrm{K}_{2}=1\right)$, on trouve la valeur du coefficient de butée $\left(\mathrm{K}_{\mathrm{p}}=4.08\right)$ correspondant au cas d'un sol homogène d'épaisseur limitée $(\mathrm{d} / \mathrm{f}=2)$. Lorsque les couches extrêmes (inférieure et supérieure) ont une perméabilité plus grande que celle de la couche intermédiaire $\left(\mathrm{K}_{1} / \mathrm{K}_{2}>1\right)$, la couche supérieure jouera le rôle de surcharge drainante augmentant la résistance en butée du sol devant la fiche (Figure 10a). Au delà d'un rapport de perméabilité égal à 10, on assiste à une diminution du coefficient de butée. Ceci s'explique par le rôle de la couche inférieure qui, du fait de sa grande perméabilité, amène, avec des pertes de charge 
négligeables, l'eau en pression sous le fond de fouille (Figure 10b), ce qui conduit ainsi à augmenter les pressions interstitielles dans la zone concernée par le mécanisme de rupture.

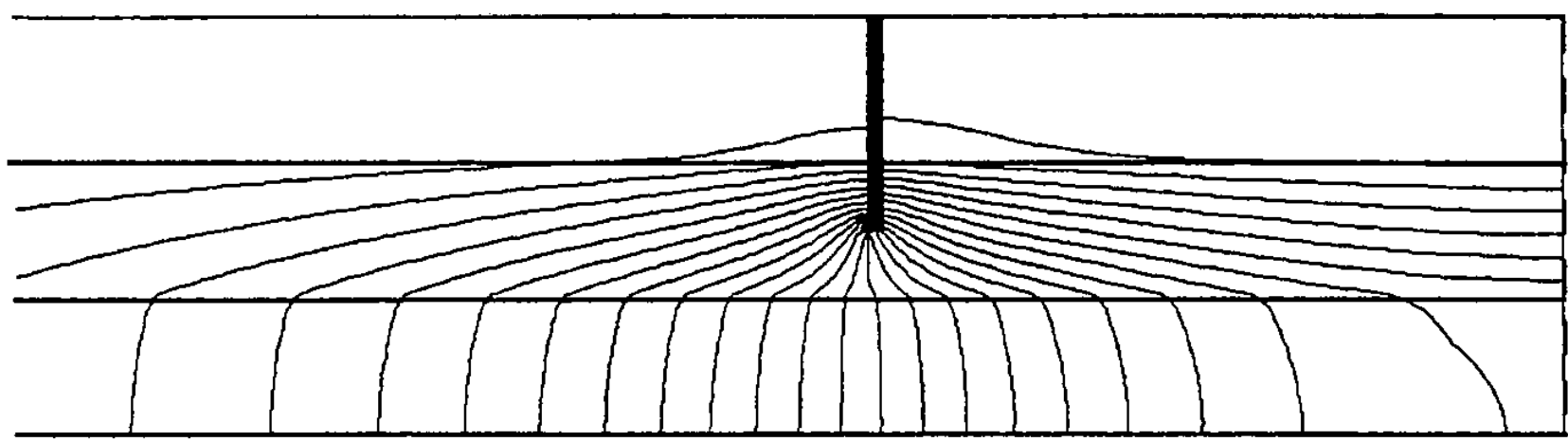

(a) $\mathrm{K}_{1} / \mathrm{K}_{2}=10$

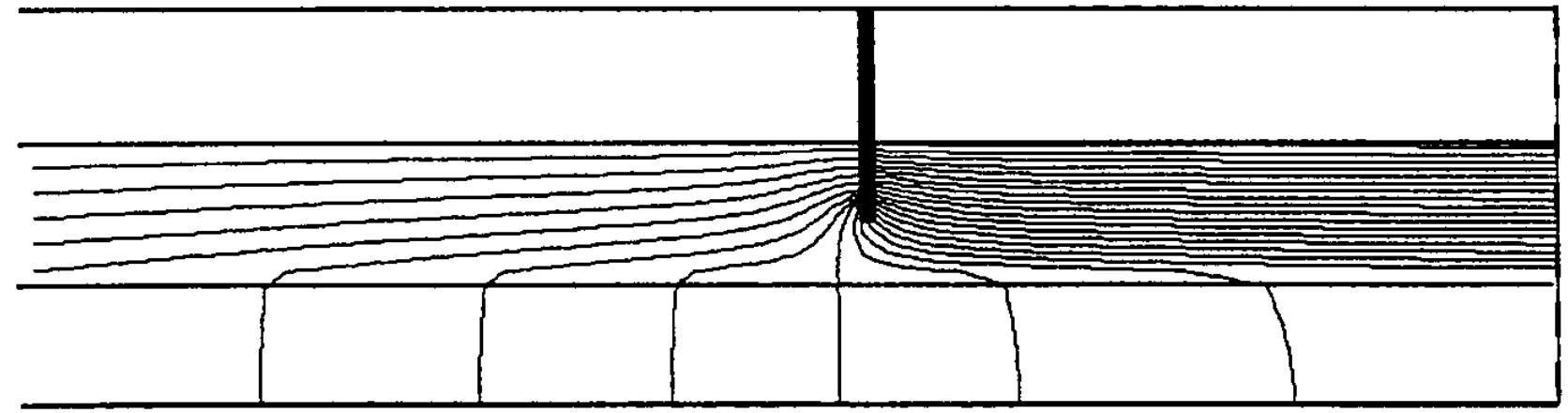

(b) $\mathrm{K}_{1} / \mathrm{K}_{2}=100$

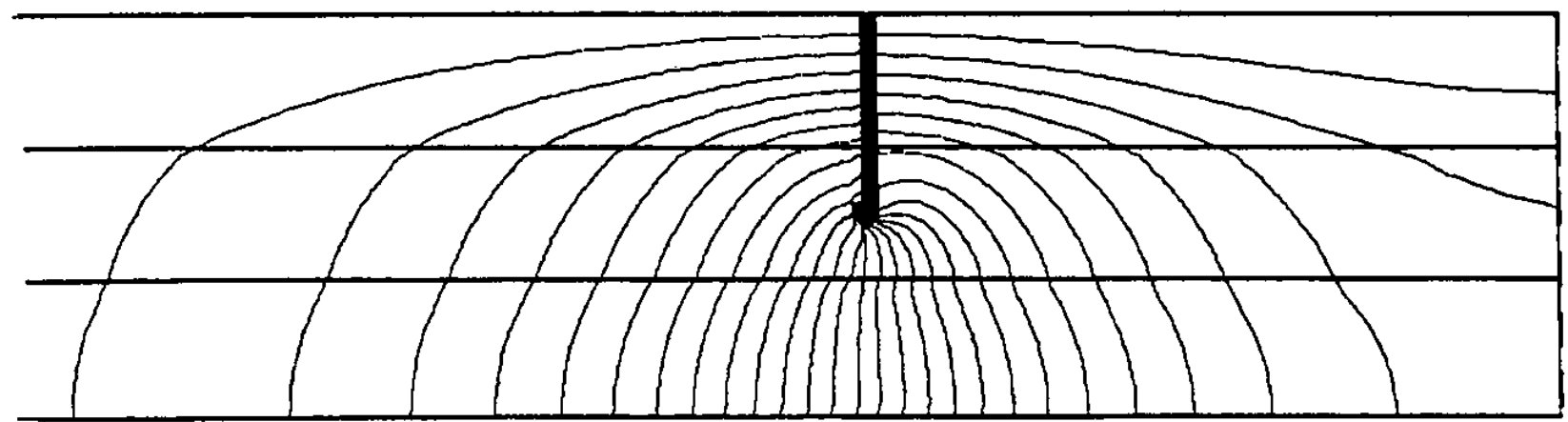

(c) $\mathrm{K}_{1} / \mathrm{K}_{2}=0.5$

Figure 10 (a, b, c). Champ des équipotentielles pour $K_{1} / K_{2}=10,100$ et 0.5 . Equipotential lines for $\mathrm{K}_{1} / \mathrm{K}_{2}=10,100$ and 0.5 
Par contre, lorsque les couches extrêmes ont une perméabilité inférieure à celle de la couche intermédiaire $\left(\mathrm{K}_{1} / \mathrm{K}_{2}<1\right)$, les pertes de charge se concentrent dans la couche supérieure (Figure 10c) : les forces d'écoulement allègent alors la couche supérieure, ce qui conduit à réduire la butée disponible. Il est à noter que le calcul numérique a montré que dans ces cas, le gradient de sortie en fond de fouille reste inférieur au gradient critique.

\section{Conclusion}

L'analyse théorique de la butée des terres en présence d'écoulement a été menée en utilisant la méthode cinématique en analyse limite et en adoptant un mécanisme de rupture rotationnel dont la ligne de glissement est décrite par une spirale logarithmique d'angle $\phi$. La comparaison des résultats numériques obtenus en l'absence d'écoulement avec ceux donnés par les tables de Caquot et Kérisel montre la pertinence du choix du mécanisme adopté. En présence d'un écoulement induit par la mise hors d'eau d'un batardeau, on a montré que la butée disponible devant la fiche de l'écran de soutènement étanche diminue avec l'augmentation de la perte de charge relative. Le cas limite d'une butée nulle représente la rupture par soulèvement généralisé d'un prisme de sol devant la fiche, souvent appelé renard. Ce mécanisme est une évolution du mécanisme simplifié proposé par Terzaghi qui supposait ce prisme rectangulaire et négligeait les forces de frottement. Le code de calcul mis au point permet de traiter des cas de sols hétérogènes et anisotropes en perméabilité. Enfin, l'étude d'un exemple d'un massif multicouche a confirmé l'importance des hétérogénéités en perméabilité vis-à-vis de la pression passive mobilisable devant la fiche d'un écran ayant simultanément un rôle de soutènement et d'étanchéité.

\section{Bibliographie}

[ANT 90] ANTHOINE, A., «Une méthode pour le dimensionnement à la rupture des ouvrages en sols renforcés », Revue Française de Géotechnique, № 50, p. 5-21, 1990.

[BAK 78] BAKER, R. \& GARber, M., "Theoretical analysis of the stability of slopes ", Géotechnique, Vol. 28, № 4, p. 395-411, 1978.

[BAS 79] Basudhar, P.K., Valsangkar, A.J. \& Madhav, M.R., «Optimal lower bound of passive earth pressure using finite elements and non-linear programming , Int. J. for Num. and Anal. Meth. in Geomech., Vol. 3, N 4, p. 367-379, 1979.

[BAZ 63] BAZANT, Z., «Ergebnisse der berechnung der stabilitat gegen hydraulischen Grundbruch mit hilfe der Elektronen-rechen anlage », Proc. Int. Conf. On Soil Mech. And Found. Engng, 24-27, p. 215-224, Budapest, 1963.

[BOT 80] Bottero, A., Nègre, R., Pastor, J., \& Turgeman, S., « Finite element method and limit analysis theory for soil mechanics problems », Computational Methods Appl. Mech. And Engng, Vol 22, p. 131-149, 1980.

[CAQ 56] Caquot, A. \& KéRISEl, J., Traité de mécanique des sols, 3rd ed., Paris : GauthierVillard, 1956. 
[CHE 73] CHEN, W.F. \& RosenFARB, J.L., «Limit analysis solutions of earth pressure problems », Soils and Foundations, Vol. 13, N 4, p. 45-60, 1973.

[CHE 75] CHEN, W.F., Limit analysis and soil plasticity, Amsterdam, Elsevier, 637 pp, 1975.

[DAV 54] DAvIDENKOFF, R.N., Zur berechnung des hydraulischen grundbruches, Wasserwirtschaft, $\mathrm{N}^{\circ} 46$, p. 230-235, 1954.

[DEB 92] De Buhan, P., Dormieux, L. \& Salençon J., « Sability analysis of reinforced soilRetaining structures using the yield design theory », Proc. Int. Conf. on Retaining Structures, 20-23 July 1992, Cambridge, England, p 1-10, 1992.

[DEB 93] De BuhAN, P., \& SALENÇON, J., « A comprehensive stability analysis of soil nailed structures », Eur. J. Mech., A/Solids, Vol 12, N³, p. 325-345, 1993.

[HAB 77] Habibagahi, K., \& Ghahramani, A., «Zero extension theory of earth pressure », J. Geotechnical Division, ASCE, Vol. 105, NGT7, p. 881-896, 1977.

[KAS 82] KASTNER, R., Excavations profondes en site urbain : problèmes liés à la mise hors d'eau. Dimensionnement des soutènements butonnés, thèse de Docteur ès Sciences, 409 pp., INSA Lyon, Université Claude Bernard Lyon I, I982.

[KER 90] KÉRISEL, J. \& ABSI, E., Tables de poussée et de butée des terres, Presses de l'Ecole Nationale des Ponts et Chaussées, $3^{\text {emc }}$ édition, 220 pp., 1990.

[LEE 72] LEE, I.K. \& HERINGTON, J.R., «A theoretical study of the pressures acting on a rigid walls by a sloping earth on rockfill », Géotechnique, Vol. 22, N 1, p. 1-26, 1972.

[LES 85] LESCHINSKY, D., BAKER, R., \& Silver, M.L., « Three-dimensional analysis of slope stability", International Joumal for Numerical and Analytical Methods in Geomechanics, Vol. 9, p. 199-223, 1985.

[LYS 70] LYSMER, J., «Limit analysis of plane problems in soil mechanics », J. Soil Mech. and Found. Div., ASCE, Vol. 96, N SM4, p. 1311-1334, 1970.

[MAN 51] MANDEL, J., «Ecoulement de l'eau sous une ligne de palplanches - Abaques pour la condition de renard », Travaux, $\mathrm{N}^{\circ} 197$, p. 273-281, 1951.

[MAR 53] MARSLAND, A., «Model experiment to study the influence of seepage on the stability of a sheeted excavation in sand », Géotechnique, Vol. 3, p. 223-241, 1953.

[PAC 69] PACKSHAw, S., « Earth pressure and earth resistance. A century of soil mechanics », the Institution of Civil Engineers, London, England, p. 409-435, 1969.

[PAS 78] PASTOR, J., "Analyse limite: Détermination de solutions statiques complètes. Application au talus vertical ", Journal de Mécanique Appliqué, Vol 2, N², p. 167-197, 1978.

[RAH 84] Rahardjo, H. \& Fredlund, D.G., «General limit equilibrium method for lateral earth force », Canadian Geotechnical Journal, Vol. 21, № 1, p. 166-175, 1984.

[SAL 83] SALENÇON J., Calcul à la rupture et analyse limite, Presse de l'ENPC, Paris, 366 pp., 1983.

[SAL 90] SalENÇON, J., «An introduction to the yield design theory and its application to soil mechanics », Eur. J. Mech., A/Solids, Vol 9, № 5, p. 477-500, 1990.

[SAL 93] SALENÇON, J., Yield design : a survey of the theory, In evaluation of the global bearing capacities of structures, ed. By G. Sacchi-Landriani, J. Salençon, SpringerVerlag, Wien, New York, 1993. 
[SHI 72] Shields, D.H. \& TolunaY, A.Z., «Passive pressure coefficients for sand by the Terzaghi and Peck method », Canadian geotechhnical Journal, Vol. 9, No 4, p. 501-503, 1972.

[SCH 73] ShIELDS, D.H., \& Tolunay, A.Z., «Passive pressure coefficients by method of slices », J. of the Geotech. Engng. Div., ASCE, Vol 99, №SM12, p. 1043-1053, 1973.

[SOK 60] SoKolovski, V.V., Statics of granular media, New-York, Pergamon Press, 270p, 1965.

[SOU 89] SoubRA, A.-H., Application de la méthode variationnelle au problème de détermination des pressions passives des terres. Influence des forces d'écoulement, thèse de Doctorat, INSA Lyon, 200pp, 1989.

[SOU 92] SOUBRA, A.-H. \& KASTNER, R., «Influence of the seepage forces on the passive earth pressures », Proc. Int. Conf. on Retaining Structures, ICE, Cambridge, p. 67-76, 1992.

[SOU 97] SouBRA, A.-H., « Seismic bearing capacity of shallow strip footings in seismic conditions », Geotechnical Engineering, The Institution of Civil Engineers, Vol. 125, $\mathrm{N}^{\circ} 4,230-241,1997$.

[SOU 98] Soubra, A.-H., Kastner, R., \& Benmansour, A., « Passive earth pressures in the presence of hydraulic gradients », Géotechnique, accepté pour publication, 1998.

[TER 43] TERZAGHI, K., Theoretical soil mechanics, 510 pp, John Wiley, New-York, 1943. 